\title{
3D Power Doppler vascular indices as a novel technique in assessing the outcome of minimally invasive techniques in uterine fibroids treatment
}

\author{
Anna Stępniak, Piotr Czuczwar \\ $3^{\text {rd }}$ Chair and Department of Gynecology, Medical University, Lublin, Poland
}

\begin{abstract}
Uterine fibroids are considered to be the most frequent female benign tumours. The most common reported symptoms of fibroids are heavy menstrual bleeding and painful menstruation, pelvic pain, urinary problems, constipation, as well as infertility and recurrent pregnancy loss. The mainstay of fibroid treatment is surgery, but nowadays minimally-invasive techniques are growing in popularity. Vascularity of fibroids may play a role in the outcome of these techniques, which is why it is important to find an objective, reproducible technique to measure the vascularization before and after the procedure. The 3D Power Doppler vascular indices (3DPDVI) allow objective assessment of vascularization in the entire volume of the tumour. Initially this technique was mostly used in experimental imaging phantoms, but recently many studies focus on the clinical utility of this technique. Power Doppler allows to obtain information on vascularity in the area of interest, while 3DPDVI can be objectively calculated by the Virtual Organ Computer-aided AnaLysis (VOCAL ${ }^{\mathrm{M}}$ ) software. 3DPDVI showed high reproducibility in most of the studies. This technique has an important role in monitoring the outcome of minimally invasive procedures in fibroid treatment, because they affect vascularity of the tumours. Although there are some limitations of 3DPDVI, it seems that their application may be an effective tool in objective assessment of vascularity of fibroids. However further studies are required to consolidate the usage of 3DPDVI in clinical practice.
\end{abstract}

Key words: vascular indices, fibroids, power Doppler, minimally invasive treatment.

\section{Introduction}

Uterine fibroids affect around half of women in the reproductive age and are considered to be the most frequent female benign tumours. The occurrence increases with age and is the highest by the menopausal age, after which it decreases constantly [1]. The symptoms of fibroids vary depending on the size and location of the tumour, however, the vast majority of the tumours are asymptomatic and are incidental findings during routine gynaecological examinations. In such cases fibroids do not require any treatment beyond observation. The most frequently reported signs and symptoms of fibroids include disturbances of the menstrual cycle such as heavy menstrual bleeding and painful menstruation, pelvic masses associated with pelvic pain, urinary problems or constipation [2].

The recommended treatment of symptomatic fibroids is myomectomy or hysterectomy, but minimally invasive alternative treatments are more available nowadays [3]. The most frequently used techniques include uterine artery embolization (UAE), magnetic resonance imaging-guided high intensity focused ultrasound (MR-HIFU), laparoscopic occlusion of uterine arteries, myolysis and ulipristal acetate (UPA) pharmacological treatment. During UAE embolic material occludes both uterine arteries, which is achieved by introducing a catheter through the femoral artery into uterine arteries under X-ray supervision. As a result, blood flow is significantly reduced and ischemia occurs in the tumour causing necrosis of fibroid cells [4]. MR-HIFU is a thermal ablation treatment modality that uses MRI guidance to deliver high intensity focused energy precisely to the target fibroid tissue, resulting in coagulative necrosis [5]. MR-HIFU is reported to be less suitable for fibroids with a high vascularity [6].

The vasculature of fibroids typically consists of a hypervascular peripheral rim and irregular central vascularization. Visualizing the vascular rim may be useful in calculating the volume of the entire fibroid and determining its location [7]. Observing vascular patterns in symptomatic uterine fibroids has resulted in the development of effective surgical and minimally-invasive techniques for the management of these tumours by reducing or eliminating their primary blood supply [8].

Vascularization plays an important role in case of minimally-invasive techniques for fibroid treatment 
and is one of the leading parameters in predicting the outcome of the therapy, which is why it was of great importance to find an objective, reproducible technique to measure the vascularization before and after the procedure. Power Doppler (PD) sonography is more sensitive and less-angle dependent than frequency-based colour Doppler sonography and more suitable in case of low blood flow and velocity [9]. PD enabled developing a new method for three-dimensional quantification of blood flow and vascularization - the cube method, which was first introduced in 1999 by Pairleitner. In this method grey-scale voxels contain all three-dimensional grey-scale information in grades from black to white. All three-dimensional colour information is summarized by colour voxels. To accentuate the difference between the amplitudes of the reflected ultrasound waves (intensity of flow), the colour voxels are weighted, i.e. multiplied by a factor from 1 (= lowest amplitude) to 32 (= highest amplitude) [10]. Pairleitner et al. proposed three 3D PD vascular indices (3DPDVI):

- vascularization index (VI) - the number of colour voxels divided by the total number of both colour and grey voxels, reflecting the proportion of blood vessels within the tissue. It is unit-less and expressed as a numerical value ranging from 0 to 100 ;

- flow index (FI) - the average colour value of all colour voxels, indicating the average flow velocity. It is unitless and expressed as a numerical value ranging from 0 to 100 ;

- vascularization-flow index (VFI) - the average colour value of all grey and colour voxels within the volume, a product of the $\mathrm{VI}$ and the $\mathrm{FI}$ (mathematical relationship derived from multiplying $\mathrm{VI}$ by $\mathrm{FI}$ and dividing the result by 100).

All calculated indices showed high reproducibility. Since Pairleitner's study more and more researchers advocated the use of 3DPDVI for blood flow mapping. The assessment of a virtually reconstructed vascular tree within a volume of interest is possible and the vascularization can be objectively estimated by calculating 3DPDVI with the use of Virtual Organ Computer-aided AnaLysis (VOCAL ${ }^{\mathrm{TM}}$ ) software [11]. Calculation of the three 3DPDVI is based on and related to the total and relative amounts of PD information within the volume of interest [12]. This method was proved to have great reproducibility ratio among ultrasonographers [13-16].

\section{Limitations of the method}

Although calculation of vascular indices with VO$C A L^{\text {TM }}$ seems to be an objective method for the assessment of fibroids' vascularization, this method also has some limitations in [17]. In most of the studies the distance between fibroids and probe was not measured, while it has been shown that 3DPDVI significantly decrease as the distance between the transducer and blood vessel increases [18]. Moreover, the result of the examination might be dependent on machine settings [19-20] and attenuation [21]. Also patients parameters such as heart pulsatility [22] and the size of the sample [23] were considered as limitations. Additionally, some authors questioned the reproducibility of 3DPDVI [24] and its clinical usefulness [25]. On the other hand, assessing changes in vascular indices obtained by the same operator on the same machine settings were pointed as a solution for at least some of the limitations [26].

\section{Liferafure review}

The number of data concerning clinical application of vascular indices is limited. Although the first reports about 3DPDVI were published some time ago, its role and clinical utility is still in research. There are more studies focusing not only on experimental phantoms, but also on patients with defined ailments.

Minsart et al. designed a study to compare 3D Doppler sonographic characteristics of fibroids with histopathological parameters of vascularization. By that time previous studies were conducted to evaluate various parameters with vascular indices, however, the correlation between ultrasonography and histopathology has not yet been studied. Seventy-three fibroids ranging from 1.4 to $8.4 \mathrm{~cm}$ in diameter were analysed first with ultrasound and then histopathologically. Interestingly there was no statistically significant relationship between 3DPDVI and patient age, smoking, parity, hormone use, black ethnicity or fibroid volume and location. However, an inverse correlation between VI and VFI and patients' BMI was shown. The study results showed that high values of VI and VFI correlated with high cellular activity scores in fibroids, while low values correlated with ischemic necrosis [27]. These findings may potentially be important in both qualifying patients to particular treatment options and monitoring their effects. Since UPA affects cellular activity and causes apoptosis while UAE results in ischaemic necrosis, it may be speculated that assessing changes in 3DPDVI may be useful in predicting the effect of these treatment options [28].

Another example of clinical usage of vascular indices was demonstrated in the study comparing vascular changes in fibroids in pregnant patients. The 3DPDVI served Degani et al. to distinguish painful leiomyomas from focal myometrial contractions and non-painful fibroids in pregnancy. The authors demonstrated that painful fibroids had significantly lower 3DPDVI than normal uterine wall. The differences in 3DPDVI between non-painful fibroids and uterine wall, as well as nonpainful fibroids and focal uterine contractions were not statistically significant [29]. 
Recently Nieuwenhuis et al. has published a series of articles focused on bypassing one of the limitations of 3DPDVI and proving that reproducibility can be achieved with satisfactory results. In their article authors reported that VI assessed using 3D PD ultrasound was a reproducible parameter in the assessment of fibroid vascularization with discriminating abilities. The researchers assessed vascular parameters such as: VI, $\mathrm{FI}$, and VFI of the entire fibroid, the vascular capsule and of its highest vascularized area, in 19 patients with symptomatic fibroids, using 3DPDVI with manual and automatic contour modes. The examinations were performed by one examiner, but evaluated by 3 independent specialists to assess reproducibility. Interobserver agreement of the vascular parameters was based on the assessment of intraclass correlation coefficients (ICCS) and their related 95\% confidence interval (CI) using a two-way mixed model. In the study all possible vascular indices of all possible relevant parts of the fibroid using both manual and automatic contour modes were calculated. The main results from the interobserver evaluation were: VI was found to be the most reproducible index, superior to $\mathrm{FI}$ and VFI. In particular, both the manually acquired VIs of the total fibroid and of the fibroid capsule showed perfect agreement. VI also showed good discriminating ability. VI was independent of fibroid volume. Vascularity of the fibroid and the capsule were correlated with each other [16]. In another study, Nieuwenhuis et al. investigated the possible limitations of 3DPDVI in the evaluation of uterine fibroid vascularization connected with ultrasound settings or cardiac cycle. 3DPDVI were obtained using three different gain settings: a fixed predetermined gain (50 dB), a higher gain $(65 \mathrm{~dB})$ and an individualised subjectively most optimal gain. Two consecutive 3D PD sweeps were taken to evaluate the effect of the cardiac cycle. The main conclusions from the study showed that predetermined fixed gain setting was not different from the individually most optimal chosen gain in vascular assessment of fibroids. A higher gain corresponded with a significantly higher VI. Potential variation during the cardiac cycle did not affect the VI in fibroids [30]. These results allow to overcome some concerns about the limitations of using 3DPDVI in gynaecology. Finally, the authors assessed the relationship between $\mathrm{VI}$ at baseline and fibroid volume, as well as, fibroid growth rate per year in 66 premenopausal women with a maximum of 2 fibroids. Achieved results were as following: all patients completed 12 months follow up without treatment. Baseline fibroid vascularisation (VI) measured with 3D PD was correlated with fibroid volume at 12 months $(p=0.02)$. An increase of $1 \%$ in $\mathrm{VI}$ at baseline was associated with a $7.00 \mathrm{~cm}^{3}$ larger fibroid volume at 12 months. Furthermore, vascularisation was also associated with fibroid growth rate per year $(p=0.04)$ [31].

There is also some data related to the usefulness of 3DPDVI in monitoring the outcome of minimallyinvasive treatment options for fibroids. Czuczwar et al. compared the effects of two minimally-invasive options for fibroid treatment: UAE and UPA, focusing on fibroid vascularity before treatment and at 3-month followup. To minimize the possible impact of fibroid location on treatment outcome, only patients with intramural fibroids were included. The VOCAL ${ }^{\text {TM }}$ programme was used to calculate VI, FI and VFI values. All 3DPDVI decreased significantly after treatment with both methods, however, the percentage of reduction in VI and VFI 3 months after UAE was significantly greater than in patients after 3 months of UPA therapy. Although two out of three measured indices decreased notably, the difference in $\mathrm{Fl}$ reduction between treatment groups did not reach significance. The authors concluded that fibroid treatment by UPA therapy resulted in fibroid vascularization decrease, however, to a lesser extent than by UAE [26]. Moreover, it was the first study, which demonstrated that a course of UPA results in a decrease in 3DPDVI. An example of 3DPDVI measured in a fibroid before and 3 months after embolization is shown on Fig. 1.
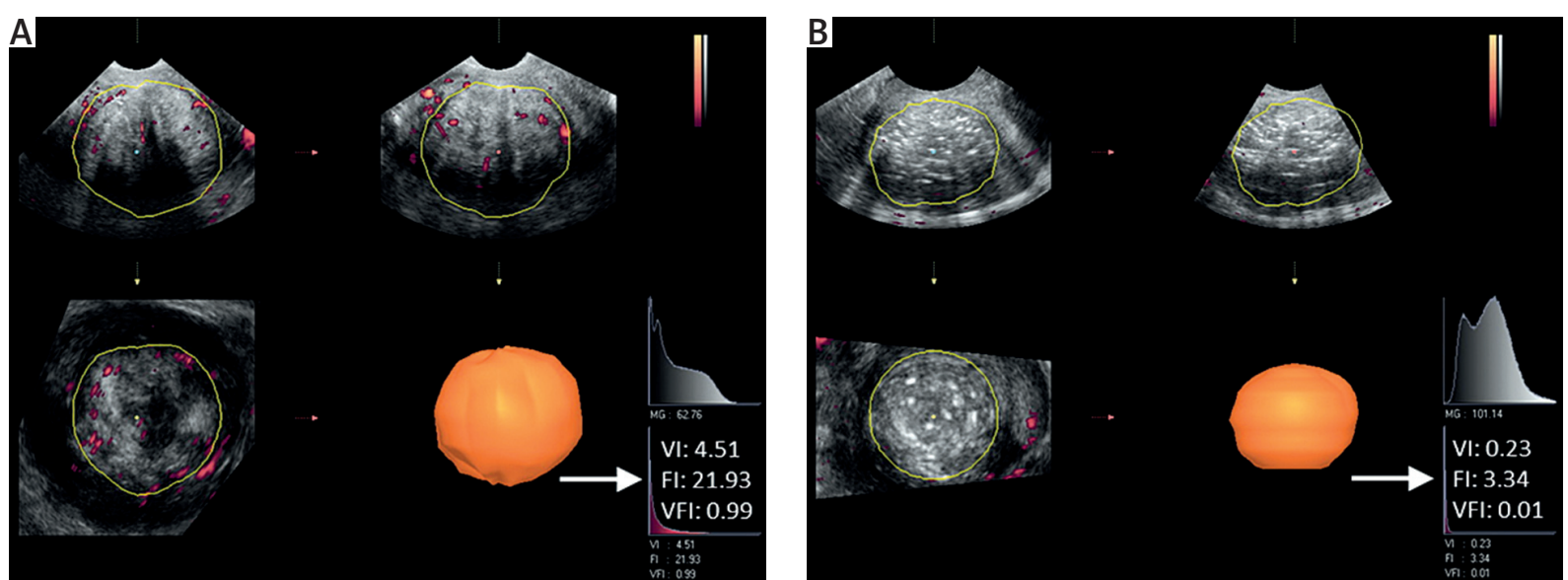

Fig. 1. 3D Power Doppler vascular indices measured before (A) and 3 months after (B) uterine artery embolization. After the embolization procedure (B) the fibroid became hyperechogenic, all vascular indices ( $\mathrm{VI}, \mathrm{FI}, \mathrm{VFI})$ decreased 


\section{Conclusions}

Available studies show that 3D PD ultrasound may be a sensitive and reliable diagnostic tool in the assessment of fibroids vasculature. This method may be used in various gynaecological fields, for example it has the potential to assess vascularity of fibroids before and after minimally-invasive procedures and may have the utility to predict the outcome of such treatment. Although further studies are required to consolidate the usage of 3DPDVI in clinical practice, the literature review has proven satisfying reproducibility of this technique. Moreover, researchers should try to overcome limitations of this method in order to allow meaningful comparison among studies. To minimize the variability of 3DPDVI international guidelines on standardized settings should be formulated.

\section{Disclosure}

Authors report no conflict of interest.

\section{References}

1. Bulun SE. Uterine fibroids. N Engl J Med 2013; 369: 1344-1355.

2. Doherty L, Mutlu L, Sinclair D, Taylor H. Uterine Fibroids. Reprod Sci 2014; 21: 1067-1092.

3. Singh SS, Belland L. Contemporary management of uterine fibroids: focus on emerging medical treatments. Curr Med Res Opin 2015; 31: 1-12.

4. Czuczwar P, Woźniak S, Szkodziak P, et al. Predicting the results of uterine artery embolization: Correlation between initial intramural fibroid volume and percentage volume decrease. Prz Menopauzalny 2014; 13: 247-252.

5. Rueff LE, Raman SS. Clinical and Technical Aspects of MR-Guided High Intensity Focused Ultrasound for Treatment of Symptomatic Uterine Fibroids. Semin Intervent Radiol 2013; 30: 347-353.

6. Funaki K, Fukunishi H, Funaki T, et al. Magnetic resonance-guided focused ultrasound surgery for uterine fibroids: relationship between the therapeutic effects and signal intensity of preexisting T2-weighted magnetic resonance images. Am J Obstet Gynecol 2007; 196: 184.e1-184.e6.

7. Kim M, Won J, Lee D, Ahn CS. Uterine artery embolization for adenomyosis without fibroids. Clin Radiol. 2004; 59: 520-526.

8. Zupi E, Piredda A, Marconi D, et al. Directed laparoscopic cryomyolysis: A possible alternative to myomectomy and/or hysterectomy for symptomatic leiomyomas. Am J Obstet Gynecol 2004; 190: 639-643.

9. Rubin JM, Adler RS, Fowlkes JB, et al. Fractional moving blood volume: estimation with power Doppler US. Radiology 1995; 197: 183-190.

10. Pairleitner H, Steiner H, Hasenoehrl G, et al. Three-dimensional power Doppler sonography: imaging and quantifying blood flow and vascularization. Ultrasound Obstet Gynecol 1999; 14: 139-143.

11. Zalud I, Shaha S. Evaluation of the utero-placental circulation by threedimensional Doppler ultrasound in the second trimester of normal pregnancy. J Matern Neonatal Med 2007; 20: 299-305.

12. Alcázar JL. Three-dimensional power Doppler derived vascular indices: what are we measuring and how are we doing it? Ultrasound Obstet Gynecol 2008; 32: 485-487.

13. Alcázar JL, Mercé LT, Manero MG, et al. Endometrial volume and vascularity measurements by transvaginal 3-dimensional ultrasonography and power Doppler angiography in stimulated and tumoral endometria: an interobserver reproducibility study. J Ultrasound Med 2005; 24: 1091-1098.

14. Arve A, Sladkevicius P, Tekay AH, et al. Intraobserver and interobserver variability of ovarian volume, gray-scale and color flow indices obtained using transvaginal three-dimensional power Doppler ultrasonography. Ultrasound Obs Gynecol 2003; 21: 277-282.

15. Raine-Fenning NJ, Campbell BK, Clewes JS, et al. The interobserver reliability of three-dimensional power Doppler data acquisition within the female pelvis. Ultrasound Obstet Gynecol 2004; 23: 501-508.
16. Nieuwenhuis LL, Betjes HE, Hehenkamp WJ, et al. The use of 3D power doppler ultrasound in the quantification of blood vessels in uterine fibroids: Feasibility and reproducibility. J Clin Ultrasound 2015; 43: 171-178.

17. Nandi A, Martins WP, Jayaprakasan K, et al. Assessment of endometrial and subendometrial blood flow in women undergoing frozen embryo transfer cycles. Reprod Biomed Online 2014; 28: 343-351.

18. Raine-Fenning NJ, Nordin NM, Ramnarine KV, et al. Determining the relationship between three-dimensional power Doppler data and true blood flow characteristics: an in-vitro flow phantom experiment. Ultrasound Obstet Gynecol 2008; 32: 540-550.

19. Miyague AH, Raine-Fenning NJ, Pavan TZ, et al. Influence of gain adjustment on 3-dimensional power Doppler indices and on spatiotemporal image correlation volumetric pulsatility indices using a flow phantom. J Ultrasound Med 2013; 32: 1831-1836.

20. Soares CA, Miyague AH, Filho FM, et al. Fractional moving blood volume (FMBV) concept can be applied to 3D power Doppler quantification. UItrasound Obstet Gynecol 2013; 41: 98-99.

21. Miyague AH, Pavan TZ, Grillo FW, et al. Influence of attenuation on three-dimensional power Doppler indices and STIC volumetric pulsatility index: a flow phantom experiment. Ultrasound Obstet Gynecol 2014; 43: 103-105.

22. Miyague a H, Raine-Fenning NJ, Polanski L, et al. Assessing repeatability of 3D Doppler indices obtained by static 3D and STIC power Doppler: a combined in-vivo/in-vitro flow phantom study. Ultrasound Obstet Gynecol 2013; 42: 571-576.

23. Martins WP, Welsh AW, Lima JC, et al. The "volumetric" pulsatility index as evaluated by spatiotemporal imaging correlation (STIC): a preliminary description of a novel technique, its application to the endometrium and an evaluation of its reproducibility. Ultrasound Med Biol 2011; 37: $2160-2168$

24. Martins WP, Lima JC, Welsh AW, et al. Three-dimensional Doppler evaluation of single spherical samples from the placenta: intra- and interobserver reliability. Ultrasound Obstet Gynecol 2012; 40: 200-206.

25. Martins WP. Three-dimensional power Doppler: validity and reliability. Ultrasound Obstet Gynecol 2010; 36: 530-533.

26. Czuczwar P, Wozniak S, Szkodziak P, et al. Influence of ulipristal acetate therapy compared with uterine artery embolization on fibroid volume and vascularity indices assessed by three-dimensional ultrasound: Prospective observational study. Ultrasound Obstet Gynecol 2015; 45: 744-750.

27. Minsart A, Ntoutoume Sima F, Vandenhoute K, et al. Does three-dimensional power Doppler ultrasound predict histopathological findings of uterine fibroids? A preliminary study. Ultrasound Obstet Gynecol 2012; 40: 714-720.

28. Park KH, Kim JY, Shin JS, et al. Treatment outcomes of uterine artery embolization and laparoscopic uterine artery ligation for uterine myoma. Yonsei Med J 2003; 44: 694-702.

29. Degani S, Tamir A., Leibovitz Z, et al. Three-dimensional power Doppler in the evaluation of painful leiomyomas and focal uterine thickening in pregnancy. Int J Gynecol Obstet. 2007; 99: 122-126.

30. Nieuwenhuis LL, Hehenkamp WJK, Brölmann HAM, et al. 3D power Doppler in uterine fibroids; influence of gain, cardiac cycle and off-line measurement techniques. J Obstet Gynaecol 2017; doi: 10.1080/01443615.2017.1330323

31. Nieuwenhuis L, Keizer A, Stoelinga B, et al. Fibroid vascularisation assessed with three-dimensional power Doppler ultrasound is a predictor for uterine fibroid growth: a prospective cohort study. BJOG An Int J Obstet Gynaecol 2017; doi: 10.1111/1471-0528.14608. 